\title{
Impact of Mesoporous Silicon Template Pore Dimension and Surface Chemistry on Methylammonium Lead Trihalide Perovskite Photophysics
}

Viviana C.P. da Costa, Roberto Gonzalez-Rodriguez, Kyle Frohna, Géraud Delport, Samuel D. Stranks, Leigh T. Canham, and Jeffery L. Coffer ${ }^{*}$

Dr. Viviana C.P. da Costa, Dr. Roberto Gonzalez-Rodriguez, and Prof. Dr. Jeffery L. Coffer Department of Chemistry and Biochemistry, Texas Christian University, TCU Box 298860, Fort Worth, Texas 76129, USA

Kyle Frohna, Dt. Géraud Delport, and Prof. Dr. Samuel D. Stranks, Cavendish Laboratory, University of Cambridge, JJ Thompson Avenue, Cambridge CB3 OHE, UK

Prof. Dr; Samuel D. Stranks

Department of Chemical Engineering and Biotechnology, University of Cambridge, Philippa Fawcett Drive, Cambridge CB3 0AS, UK

Prof. Dr; Leigh T. Canham

Nanoscale Physics, Chemistry, and Engineering Research Laboratory, University of Birmingham, Birmingham, B15 2TT UK

Keywords: Perovskite, photoluminescence, defects, porous silicon, interfaces, photovoltaics

In influencing fundamental properties—and ultimately device performance—of lead halide perovskites, interfacial interactions play a major role, notably with regard to carrier diffusion and recombination. Here, anodized porous $\mathrm{Si}(\mathrm{pSi})$ as well as porous silica 
particles are employed as templates for formation of methylammonium lead trihalide nanostructures. This allows synthesis of relatively small perovskite domains and comparison of associated interfacial chemistry between as-prepared hydrophobic hydride-terminated functionalities and hydrophilic oxide-terminated surfaces. While physical confinement of $\mathrm{MAPbBr}_{3}$ has a uniform effect on carrier lifetime, pore size of the silicon-containing template $(7-18 \mathrm{~nm})$ has a sensitive influence on resultant perovskite photoluminescence (PL) wavelength maximum. Furthermore, identity of the surface functionality of the template significantly alters the PL quantum efficiency, with lowest PL intensity associated with the H-terminated $\mathrm{pSi}$ and the most intense PL affiliated with oxide-terminated $\mathrm{pSi}$. These effects are explored for green-emitting $\mathrm{MAPbBr} 3$ as well as infrared-emitting $\mathrm{MAPbI}_{3}$. In addition, the role of silicon surface chemistry on the time-dependent stability of these perovskites packaged within a given mesoporous template is also evaluated, specifically, a lack of miscibility between MAPbI3 and the H-terminated pSi template results in a diffusion of this specific perovskite composition from this porous matrix over time.

\section{Introduction}

Hybrid organic-inorganic metal halide perovskites continue to be investigated for

promising performance in photovoltaic ${ }^{[1-5]}$ and optoelectronics ${ }^{[6-12]}$ applications, with their electronic and optical properties readily tuned by changing perovskite composition and size. Perovskite structures are of the composition $\mathrm{ABX}_{3}$, where $\mathrm{A}$ is typically an organic (e.g. methylammonium, $\mathrm{CH}_{3} \mathrm{NH}_{3}{ }^{+}$(MA), formamidinium, $\mathrm{CH}\left(\mathrm{NH}_{2}\right)_{2}{ }^{+}$(FA)) or 
inorganic cation (e.g. $\left.\mathrm{Cs}^{+}\right), \mathrm{B}$ a metal cation (e.g. $\left.\mathrm{Pb}^{2+}, \mathrm{Sn}^{2+}\right)$, and $\mathrm{X}$ a halide anion $\left(\mathrm{I}^{-}, \mathrm{Br}^{-}\right.$ , $\left.\mathrm{Cl}^{-}\right)$. Binary or ternary mixtures of these cations or halides can also be used to obtain more complex and chemically diverse perovskite structures, with compositional optimization (and other design features) leading to perovskite solar cell power conversion efficiencies higher than $25 \% \cdot{ }^{[13]}$ Such competitive performance features are a consequence of a number of useful fundamental properties: a high absorption coefficient, ${ }^{[14]}$ long charge-carrier diffusion length, ${ }^{[15]}$ tunable bandgap, ${ }^{[16]}$ low exciton binding energy, ${ }^{[17]}$ high charge-carrier mobility, ${ }^{[18]}$ and small Urbach band tail energy. ${ }^{[19]}$

It is clear from a number of reports that interfacial interactions play a key, sensitive role in affecting the photophysical properties of these perovskites, notably with regard to carrier diffusion and recombination. ${ }^{[20]}$ One approach to probe the influence of these interfacial interactions is to deliberately form a given perovskite within a porous templating solid. ${ }^{[21-28]}$ In terms of device compatibility / integration, templates based on elemental silicon-based materials are an appealing option to investigate given their widespread use in tandem solar cell designs. ${ }^{[29]}$ Previous studies from our group have focused on the use of hollow silicon nanotubes of relatively large inner diameter (30 to $200 \mathrm{~nm})$ to direct perovskite formation. ${ }^{[25-28]}$ In this work, we employ anodized $\mathrm{Si}$ particles ${ }^{[30]}$ as templates with pore sizes in the mesoporous range (average pore diameter $7-18 \mathrm{~nm}$ ) to access small perovskite domains and also gain the ability to switch surface chemistry between an as-prepared hydrophobic hydride-terminated functionality ${ }^{[31]}$ and a hydrophilic oxide-terminated one. It is found that while physical confinement of $\mathrm{MAPbBr}_{3}$ has a uniform effect on carrier lifetime, the pore size of the silicon-containing template has a sensitive influence on resultant perovskite photoluminescence (PL) 
wavelength maximum, and the identity of surface functionality of the template significantly alters the emission intensity. We explore these effects for the case of greenemitting $\mathrm{MAPbBr}_{3}$ as well as the infrared-emitting $\mathrm{MAPbI}_{3}$. Finally, the rather pragmatic issue of the time-dependent stability of these perovskites packaged within a given mesoporous template is also addressed. Stored in air, $\mathrm{MAPbBr}_{3}$ nanostructures formed within pSi templates gradually lose emission intensity as a function of time, but the behavior of $\mathrm{MAPbI}_{3}$ formed within the hydride-terminated porous $\mathrm{Si}$ template is significantly different, as it diffuses from the pSi matrix and actually gains PL intensity over time, a consequence of immiscible interfacial chemistry with the host matrix.

\section{Results and Discussion}

In this work, the formation of two different perovskite nanostructure compositions, $\mathrm{CH}_{3} \mathrm{NH}_{3} \mathrm{PbX}_{3}(\mathrm{X}=\mathrm{Br}$, I) is described using porous silicon-based templates to dictate the size of the resulting perovskite. Multiple silicon-based templates with different surface chemistries and pore size were selected, specifically hydrogenterminated mesoporous silicon $(\mathrm{pSi}-\mathrm{H})(2$ types, average pore size $=10 \mathrm{~nm}$ and $18 \mathrm{~nm})$, oxygen-terminated porous silicon (pSi-Ox) $(3$ types, average pore size $=10 \mathrm{~nm}, 17 \mathrm{~nm}$, and $18 \mathrm{~nm}$ ) and mesoporous silica $\left(\right.$ meso- $\left.\mathrm{SiO}_{2}\right)$ (average pore size $=7 \mathrm{~nm}$ ). Silicon content, percent of oxidation and pore size for the different types of template materials used in this work are outlined in Table 1 (along with sample ID), with silicon content and percent oxidation determined by energy dispersive x-ray (EDX) analyses. It should also be noted that the identity of such surface functionalities was confirmed for a given type of pSi sample via FT IR (Supporting Information, Fig S4) 
Table 1: Template material properties: silicon content, percent of oxidation and average pore size.

\begin{tabular}{|c|c|c|c|}
\hline Sample ID & Si Content (Atomic \%) & $\begin{array}{c}\text { O Content } \\
\text { (Atomic \%) }\end{array}$ & $\begin{array}{c}\text { Pore } \\
\text { Size } \\
\text { (nm) }\end{array}$ \\
\hline Meso-SiO 2 & $33.40 \pm 2.15$ & $66.60 \pm 2.15$ & 7 \\
\hline pSi-H-1 & $85.79 \pm 7.52$ & $14.21 \pm 7.52$ & 10 \\
\hline pSi-Ox-1 & $50.32 \pm 6.64$ & $49.69 \pm 6.64$ & 10 \\
\hline pSi-Ox-2 & $33.30 \pm 3.04$ & $66.70 \pm 3.04$ & 17 \\
\hline pSi-H-3 & $89.15 \pm 2.10$ & $10.85 \pm 2.10$ & 18 \\
\hline pSi-Ox-3 & $38.30 \pm 3.42$ & $61.70 \pm 3.42$ & 18 \\
\hline
\end{tabular}

Transmission electron microscopy (TEM) was used to analyze the structures of $\mathrm{MAPbBr}_{3}$ and $\mathrm{MAPbI}_{3}$ perovskite nanocrystals embedded in the porous silicon-based templates. In

Figure 1, TEM images of $\mathrm{MAPbBr}_{3}$ formed inside the porous structure of

mesoporous $\mathrm{SiO}_{2}$ (Figure 1a and 1d), pSi-Ox-2 (Figure 1b), and pSi-H-1 (Figure 1c) are shown. TEM images reveal the presence of spherical or slightly ellipsoidal perovskite nanostructures with a size smaller than the average pore size of a given template material, with perovskite crystallite size usually in the 4 to $9 \mathrm{~nm}$ range. Analysis of the observed lattice spacings at high resolution are consistent with the (200) and (211) crystal planes of this perovskite. ${ }^{[32]}$ Representative X-ray diffraction (XRD) measurements performed on freshly-prepared $\mathrm{MAPbBr}_{3}$ perovskite impregnated templates (Supporting Information, Figure S1) show that the $\mathrm{MAPbBr}_{3}$ perovskite-associated peaks are consistent with the presence of the cubic phase. ${ }^{[32]}$ No peaks associated with pure $\mathrm{PbBr}_{2}$ and MABr phases were found. Selected TEM images for $\mathrm{MAPbI}_{3}$ formed inside porous silicon-based templates are shown in Supporting Information (Figure S2), with discrete nanocrystals exhibiting the expected lattice spacings for the tetragonal phase clearly present. ${ }^{[33]}$ 


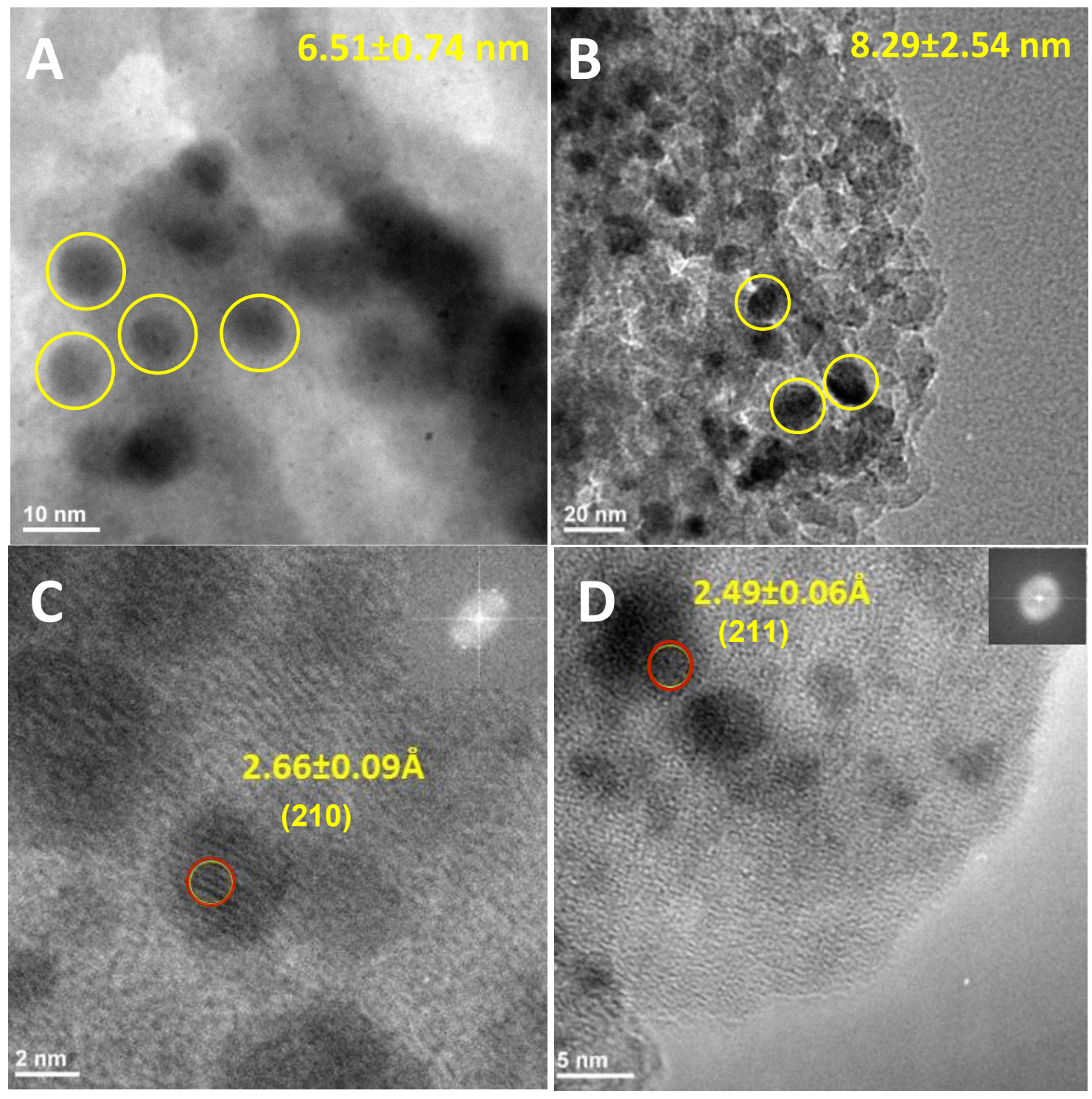

Figure 1: TEM images of templates filled with $\mathrm{MAPbBr}_{3}$ : (a) meso- $\mathrm{SiO}_{2}$; (b) pSi-Ox-2, (c) pSi-H-1, (d) meso-SiO ${ }_{2}$. In $\mathrm{A}$ and $\mathrm{B}$, the presence of discrete perovskite nanocrystals is highlighted with yellow circles, with average nanocrystal diameter listed; in $\mathrm{C}$ and $\mathrm{D}$, red circles highlight lattice spacings in selected nanocrystals, with corresponding Miller indices of $\mathrm{MAPbBr}_{3}$ labelled.

Here we investigate how both the luminescence intensity and emission wavelength maximum of the perovskite phases formed within the above pSi materials are sensitive to template surface functionalization, template pore size, and perovskite 
composition. We begin with a discussion of the role of template surface functionality on perovskite emission intensity. Figure $2 \mathrm{a}$ shows a series of photoluminescence (PL) spectra for $\mathrm{MAPbBr}_{3}$ formed within: mesoporous silicon (pSi, average pore diameter 10 $\mathrm{nm}$ ) that possesses either (a) hydride or (b) oxide surface functionalities; (c) mesoporous silica (average pore diameter $7 \mathrm{~nm}$ ) templates, as well as (d) bulk microcubes of this same perovskite composition. It is clear that the $\mathrm{MAPbBr}_{3}$ formed within the mesoporous silica possesses the strongest PL intensity of the templated systems shown, followed by this same perovskite formed within the oxide terminated $\mathrm{pSi}$, with the emission from the perovskite with the silicon hydride terminated interface exhibiting the relatively weakest emission associated with the $\mathrm{MAPbBr}_{3}$.

These differences are quantified in terms of measurements of PL quantum efficiency (PLQE) for $\mathrm{MAPbBr}_{3}$ formed within these three host matrices (PLQE: meso$\mathrm{SiO}_{2}>$ oxidized $\mathrm{pSi}>\mathrm{H}$-terminated $\mathrm{pSi}$; Table 2). The relatively strongest PL for $\mathrm{MAPbBr}_{3}$ formed within mesoporous $\mathrm{SiO}_{2}$ is attributed to an enhanced radiative character associated with the quasi-confined $6 \mathrm{~nm}$ perovskite particles ${ }^{[34]}$ (Fig 1a) as well as some passivation from the oxide moieties of the silica template. ${ }^{[35-378]}$ The perovskite formed within the larger average pore diameter $(10 \mathrm{~nm})$ of the pSi framework yields a slightly larger perovskite nanocrystal average size (Fig 1b), and the greater PL intensity of $\mathrm{MAPbBr}_{3}$ templated by the surface oxidized pSi over that of the silicon hydride is attributed to known passivation of shallow traps near the conduction band edge by oxospecies. $^{[35]}$ 
In addition to differences in oxide content (evaluated via FT IR, Supporting Information, Fig S4), energy dispersive x-ray analysis (EDX) confirms the significantly greater Si content in the hydride-terminated pSi host matrix (Table 1). A similar trend is observed in the relative PL intensity differences between $\mathrm{MAPbI}_{3}$ housed within oxideterminated $\mathrm{pSi}$ and hydride-terminated $\mathrm{pSi}$ (with the oxide terminated interface more luminescent), albeit with a smaller difference between the oxide-hydride surfaces than in the case of the pair of bromide-containing perovskite interfaces (Fig 2b).
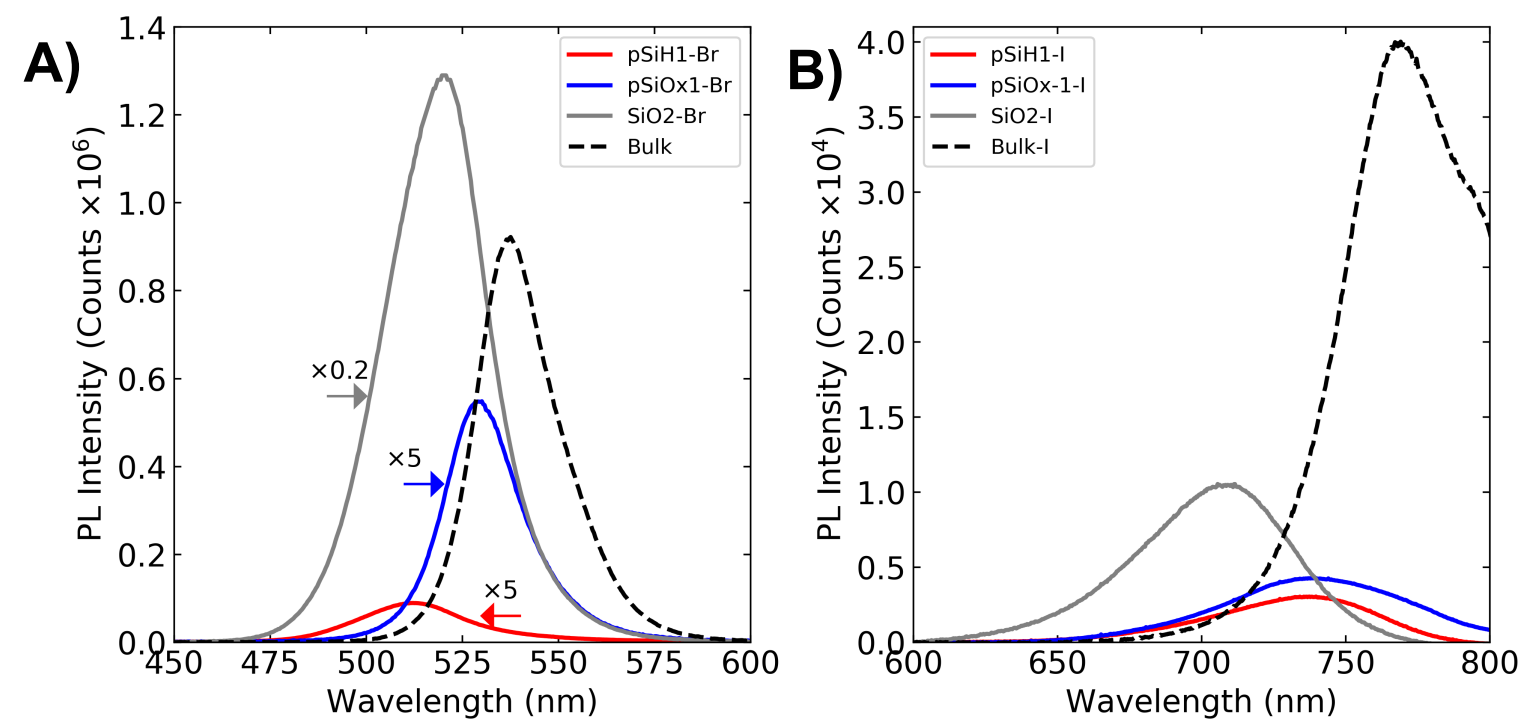

Figure 2: Room-temperature PL spectra for: (A) $\mathrm{CH}_{3} \mathrm{NH}_{3} \mathrm{PbBr}_{3}$ formed as bulk (dashed line) and inside mesoporous pSi-H-1 (red line), $\mathrm{pSi}-\mathrm{Ox}-1$ (blue line) and $\mathrm{SiO}_{2}$ (grey line); (B) $\mathrm{CH}_{3} \mathrm{NH}_{3} \mathrm{PbI}_{3}$ formed as bulk (dashed line) and inside mesoporous $\mathrm{pSi}-\mathrm{H}-1$ (red line), pSi-Ox-1 (blue line) and $\mathrm{SiO}_{2}$ (grey line). Emission peak maxima are explicitly identified on the basis of maximum peak intensity. 
Table 2: PL Quantum efficiency (PLQE) of $\mathrm{MAPbBr}_{3}$ perovskites confined within silicon-based mesoporous materials.

\begin{tabular}{|c|c|c|c|}
\hline Material & O/Si Atomic Ratio & Pore Size (nm) & QE (\%) \\
\hline SiO $_{2}$ & 1.994 & 7 & $2.87( \pm 0.64)$ \\
\hline pSi-Ox-1 & 0.987 & 10 & $0.067( \pm 0.003)$ \\
\hline pSi-H-1 & 0.166 & 10 & $<<0.02$ \\
\hline
\end{tabular}

We now analyze the impact of average pore diameter on $\mathrm{MAPbX}_{3}$ emission wavelength maximum. In general, physical confinement of the perovskite within the template is associated with a clear blue shift in wavelength peak with respect to the bulk phase for both $\mathrm{MAPbBr}_{3}$ and $\mathrm{MAPbI}_{3}$. For the $\mathrm{MAPbBr}_{3}$ system in both the oxideterminated $\mathrm{pSi}$ and hydride-terminated $\mathrm{pSi}$, there is a sensitive trend as the wavelength peak shifts to the blue with decreasing pore size (Figure 3). This shift is consistent with the presence of quasi size-dependent quantum size effects in the perovskite nanocrystals formed, as the mean nanocrystal size of $\mathrm{MAPbBr}_{3}$ is influenced by decreasing pore diameter of the template (Fig 1). Previous accounts have documented such shifts for $\mathrm{MAPbBr}_{3}$ nanocrystals in the size regime observed here. ${ }^{[32,34,38]}$

For $\mathrm{MAPbBr}_{3}$ stabilized by comparably-sized template diameters of $10 \mathrm{~nm}$, the emission maximum of the hydride terminated interface is blue shifted by $\sim 0.1 \mathrm{eV}$ relative to the oxide-terminated one. There are two possible explanations for this effect: (a) perovskite size. Limited TEM analysis of $\mathrm{MAPbBr}_{3}$ nanocrystals formed within the two matrices reveal a slightly smaller average nanocrystal size for $\mathrm{MAPbBr}_{3}$ formed within the H-terminated pSi interface $(3.7+0.9 \mathrm{~nm})$ compared to the oxide-terminated interface $(4.3+0.4 \mathrm{~nm})($ Fig S5). This is likely a consequence of the lack of chemical wettability between the hydrophobic H-termination ${ }^{[31]}$ and the perovskite; (b) differences 
in the local dielectric environment between a given perovskite and oxide-terminated surface and hydride are also a consideration in this case. ${ }^{[40]}$ It should be emphasized, however, that the above-stated size differences between perovskite nanocrystals are not statistically different.
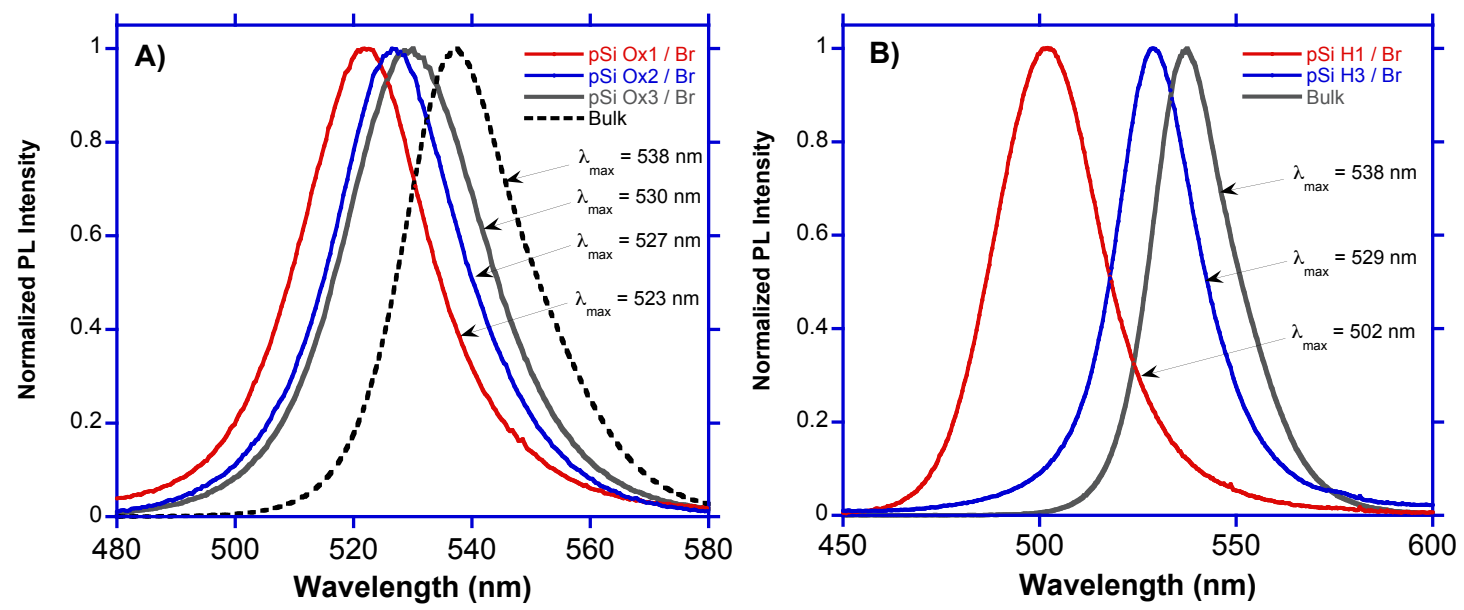

Figure 3: Normalized room-temperature $\mathrm{PL}$ spectra for $\mathrm{CH}_{3} \mathrm{NH}_{3} \mathrm{PbBr}_{3}$ formed inside mesoporous silicon templates as a function of template pore size: (A) pSi-Ox-1 $(10 \mathrm{~nm}$, red line), pSi-Ox-2 (17 nm, blue line) and pSi-Ox-3 (18 nm, grey line); (B) pSi-H-1 (10 $\mathrm{nm}$, dash-dot line) and pSi-H-3 (18 nm, full line). $\mathrm{PL}$ spectrum for $\mathrm{CH}_{3} \mathrm{NH}_{3} \mathrm{PbBr}_{3}$ bulk is presented for comparison (dashed line for A; solid line for B).

In addition to the PLQE measurements noted above, we also measured the average PL decay lifetime of the $\mathrm{MAPbBr}_{3}$ nanostructures encapsulated in these mesoporous templates, evaluated microscopically. A typical PL lifetime map for $\mathrm{MAPbBr}_{3}$ housed within a hydride-terminated $\mathrm{pSi}$ template is shown in Figure 4 (representative data for other sample types are found in Supporting Information, Figure S8), and the range of lifetimes measured for a given sample type is summarized in Table 3. 

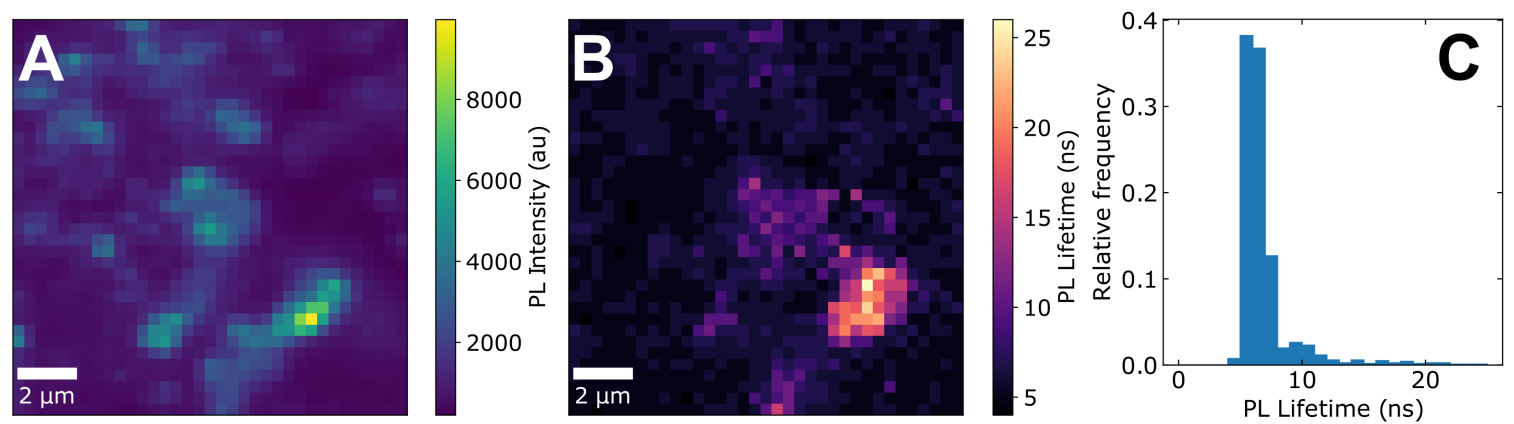

Figure 4. Confocal PL intensity map (a) and lifetime map (b) for $\mathrm{MAPbBr}_{3}$ housed within hydride-terminated pSi (10 nm ave diameter, pSi-H1). (c) Histogram of PL lifetimes extracted from panel (b). Note: local lifetimes are defined as the time taken to fall to $1 / \mathrm{e}$ of the initial intensity for each decay.

Table 2. Average PL lifetime range (ns) for $\mathrm{MAPbBr}_{3}$ formed within a given porous template (room temperature).

\begin{tabular}{|c|c|}
\hline Template & PL Lifetime Range (ns) \\
\hline $\mathrm{pSi}-\mathrm{Ox}-1$ & $5.7 \pm 1.8$ \\
\hline $\mathrm{pSi}-\mathrm{H}-1$ & $5.5 \pm 2.3$ \\
\hline $\mathrm{Meso}-\mathrm{SiO}_{2}$ & $7.5 \pm 3.3$ \\
\hline
\end{tabular}

For all samples, the observed mean lifetimes fall within the 5-8 ns range, significantly reduced from the non-templated perovskite lifetimes that are typically an order of magnitude longer. ${ }^{[32,38]}$ Such a reduction ${ }^{[39]}$ is consistent with an increased rate of radiative recombination contribution, likely influenced by two factors: 1) significant local increases in carrier densities in the nanoscale perovskite (carriers are inhibited from diffusing away); along with: 2) effect of refractive index increases with the presence of the perovskite inside the mesoporous silicon templates. ${ }^{[40]}$ The observation that the lifetime is very similar for all three samples, even though their corresponding PLQE values change by 2 orders of magnitude (Table 2) points to much of the recombination 
being faster than our system resolution, i.e. much of the radiative fraction happens in the sub $100 \mathrm{ps}$ regime, and we probe a tail that is (relatively) common to each.

Complementing all of the above measurements is the logical question of how a given nanoporous template impacts the long term photophysical stability of the perovskite, as evaluated in terms of time-based spectral evolution of $\mathrm{MAPbX}_{3} \mathrm{PL}$. Previous measurements for mixed halides formed within different inner diameter silicon nanotube templates suggest differences in PL and phase stability as a function of template diameter, with decreasing stability associated with decreasing template diameter (for NTs of 30, 70, and $200 \mathrm{~nm}$ diameter). ${ }^{[25]}$

For the $\mathrm{MAPbBr}_{3}$ templated by the mesoporous materials evaluated in this study (ranging from 7 to $18 \mathrm{~nm}$ in diameter, a significantly smaller range than the SiNTs evaluated previously), significant loss of PL intensity (70-90\%) occurs within a two-week period, in a manner relatively insensitive to template identity (Supporting Information Fig S6). For all perovskite samples, this decrease in intensity is associated with perovskite degradation due to oxygen and humidity exposure, ${ }^{[41]}$ as these perovskites are prepared / measured in ambient laboratory air.

However, there is a distinctive difference in the interfacial stability between the above $\mathrm{MAPbBr}_{3}$ structures and the corresponding time-based spectral evolution of $\mathrm{MAPbI}_{3}$ perovskites formed within these different mesoporous silicon/silica particle templates. While significant PL degradation is observed for $7 \mathrm{~nm}$ diameter mesoporous silica impregnated with $\mathrm{MAPbI}_{3}$ after 2 weeks $(\sim 90 \%$, Figure $5 \mathrm{a})$, and a smaller diminution for the $\mathrm{MAPbI}_{3}$ templated by pSi-Ox-1 $(\sim 30 \%$, Figure $5 b)$, a rather radically different spectral evolution behavior takes place for $\mathrm{MAPbI}_{3}$ templated by $\mathrm{pSi}-\mathrm{H}-1$ (10 
nm ave. pore d). After only 1 week, the perovskite emission peak shifts from an initial value of ca. $735 \mathrm{~nm}$ to an emission maximum at $765 \mathrm{~nm}$ (the characteristic peak location for bulk $\mathrm{MAPbI}_{3}$ perovskite). Furthermore, the appearance of this new peak is coupled with a significant increase in intensity (Figure 5c). The structural origin of this spectral evolution is evaluated by comparing SEM images of these materials recorded immediately after sample preparation and after aging for 2 weeks. While no perovskite precipitation is observed in freshly prepared samples (Figure 5d), the presence of perovskite crystals at the surface of the hydride-terminated pSi template is quite evident (Figure 5e). The migration of perovskite from inside the template pores to its outer surface likely originates from the strong hydrophobic character of the hydride terminated pSi surface, coupled with its interaction with the soft, polarizable iodide-containing perovskite phase. It is likely that some residual hydrophilic DMF solvent is trapped within the $\mathrm{MAPbI}_{3}$ encapsulated in the hydrophobic porous silicon, thereby accentuating the diffusion of the perovskite from the nanoporous network over time. Consistent with this observation is a relative increase, after ageing one week, in light emission associated with the $\mathrm{MAPbI}_{3}$ imaged directly with a PL microscope (Fig 5f, g). 


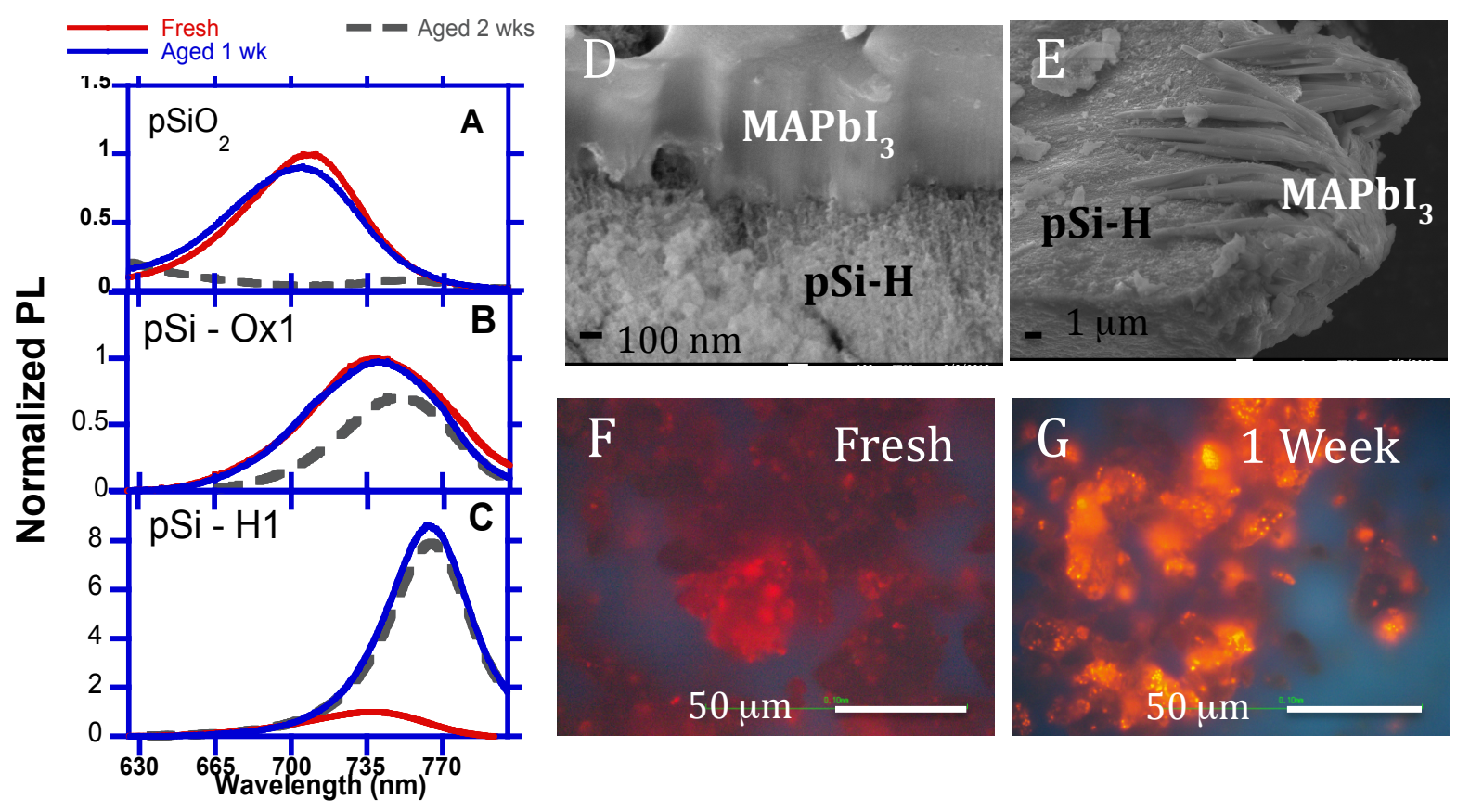

Figure 5: Evolution of PL spectra (up to two weeks) for $\mathrm{CH}_{3} \mathrm{NH}_{3} \mathrm{PbI}_{3}$ perovskites formed within (a) mesoporous silica $\left(\mathrm{pSiO}_{2}\right)$, (b) $\mathrm{pSi}-\mathrm{Ox}-1$ and (c) $\mathrm{pSi}-\mathrm{H}-1$. All spectra were acquired at room temperature and are normalized with respect to freshly (time $=0$ ) prepared samples. (d)-(e) FE-SEM images of $\mathrm{CH}_{3} \mathrm{NH}_{3} \mathrm{PbI}_{3}$ perovskites formed within pSi-H-1 taken after 1 week. (f)-(g) PL microscopic images of pSi-H-1 impregnated with $\mathrm{CH}_{3} \mathrm{NH}_{3} \mathrm{PbI}_{3}$ (f) immediately after being prepared and $(\mathrm{g})$ after 1 week of storage in the desiccator. Red emission in each image is associated with the presence of $\mathrm{MAPbI}_{3}$.

Once eluted from the pSi network, the degradation of the perovskite PL occurs in a rather heterogeneous manner. This is most aptly visualized in the case of bulk $\mathrm{MAPbI}_{3}$ samples of a microwire morphology, where the average emission intensity degrades slightly over a three-week period, but the standard deviation associated with such measurements increases several fold (Supporting Figure S7). The underlying cause for this effect can be directly visualized in a comparison of relatively large area fluorescence images between freshly prepared $\mathrm{MAPbI}_{3}$ and a three-week aged sample of the same composition (Supporting Figure S7). For the latter, it is clear that bright red emissive 
spots are present, but the amount of dark, non-luminescent regions has increased. Thus while not totally suppressing degradation of a given perovskite phase, the template does play a role in mediating the process.

\section{Conclusion}

These results establish the sensitive dependence of lead halide perovskite photophysics on the size, composition, and surface chemistry associated with the host porous silicon-based template with which it interacts. Specifically, use of such relatively small pore diameter templates dictate the formation of quasi-quantum confined nanocrystals of $\mathrm{MAPbX}_{3}$ whose emission maxima wavelength is correlated with template diameter. Associated PL lifetime measurements are on the order of several nanoseconds, consistent with increased carrier densities in restricted diffusion paths. Importantly, the perovskite emission intensity is sensitively influenced by the chemical functionality at the surface of the porous silicon template. Further development of our fundamental understanding of such properties has significant implications for perovskite-based applications, most notably in the optimal performance of tandem devices of diminishing dimension containing these two entities. Experiments providing such information are underway.

\section{Experimental}

Materials. Lead bromide $\left(\mathrm{PbBr}_{2}, \quad \geq 98 \%\right)$, lead iodide $\left(\mathrm{PbI}_{2}, \quad 99 \%\right), \quad \mathrm{N}, \mathrm{N}-$ dimethylformamide (DMF, $\geq 99.7 \%)$ and mesoporous silica powder $\left(\mathrm{SiO}_{2}, \mathrm{~d}_{\text {pore }} \approx 7.1 \mathrm{~nm}\right)$ were purchased from Sigma-Aldrich. Methylammonium bromide (MABr) and 
methylammonium iodide (MAI) were acquired from Greatcellsolar. All of the above chemicals were used without further purification.

Preparation of Porous Silicon (pSi) particles. pSi membranes possessing a mesoporous morphology were prepared by electrochemical anodization of $\mathrm{Si}<100>$ substrates in methanolic HF electrolyte to create $150 \mu \mathrm{m}$ thick membranes of $81 \%$ porosity. The membranes were mechanically ground by hand and sieved to a particle size range of $38-$ $75 \mu \mathrm{m}$. For selected experiments, pSi particles were surface oxidized by a 60 min anneal in air at $600^{\circ} \mathrm{C}$ in order to convert the pSi surface from a hydrophobic to hydrophilic one. Mean pore size for a given pSi sample was dictated by magnitude of applied bias during the above anodization experiments, and determined experimentally by low temperature nitrogen adsorption measurements subsequently fit to a Brunauer-Emmitt-Teller (BET) isotherm. ${ }^{[41]}$ Surface area, pore volume, and pore diameter values derived from these measurements for the various samples utilized in the experiments described herein are provided in Supporting Information (Table S1). It should be noted that all of the $\mathrm{pSi}$ templates utilized in these experiments (both hydride and oxide terminated) lack intrinsic detectable visible PL.

Preparation of Perovskite Precursor Solutions. Perovskites of $\mathrm{CH}_{3} \mathrm{NH}_{3} \mathrm{PbX}_{3}(\mathrm{X}=\mathrm{Br}, \mathrm{I})$ were prepared by dissolving equimolar amounts of methylammonium bromide (or iodide) and lead bromide (or iodide) in DMF solvent (200 mM) at room temperature. Solutions were sonicated for 5 to $10 \mathrm{~min}$, followed by vortex stirring to ensure complete dissolution and mixing. This prepared transparent solution was used as is for the preparation of silicon-perovskite based materials as well as bulk perovskite phases (as controls). 
Preparation of Bulk Perovskite Structures. Perovskite bulk structures were prepared by placing $\sim 0.1 \mathrm{~mL}$ of a perovskite precursor solution (at $200 \mathrm{mM}$ concentration) on a piece of FTO glass. The sample on the FTO glass was then heated for 30 min at $95{ }^{\circ} \mathrm{C}$. Characterization was performed on freshly prepared samples and aged samples kept in a desiccator.

Preparation of Silicon Perovskite-Containing Materials. In a given experiment, $1 \mathrm{~mL}$ of perovskite precursor solution at $200 \mathrm{mM}$ concentration was added to $10 \mathrm{mg}$ of mesoporous silicon (or mesoporous $\mathrm{SiO}_{2}$ ) powder. This mixture was then heated at 65-70 ${ }^{\circ} \mathrm{C}$ under stirring for $4 \mathrm{~h}$ for complete impregnation of the precursor solution within the silicon (or $\mathrm{SiO}_{2}$ ) mesoporous structure. Afterwards, the mixture was centrifuged for approximately $3 \mathrm{~min}$. The supernatant liquid was removed using a micropipette and the remaining silicon (or $\mathrm{SiO}_{2}$ ) powder collected using a spatula. The as-obtained powder was then sandwiched between two microscope glass plates and heated at $95{ }^{\circ} \mathrm{C}$ in an oven for $30 \mathrm{~min}$. The resulting perovskite-impregnated powder was then placed under overnight vacuum to assure complete solvent removal. The freshly obtained materials are then characterized. After initial characterization, these materials are stored and aged in a desiccator and used for further analyses.

Material Characterization. Transmission electron microscopy (TEM) images were obtained using a JEOL JEM-2100, operating at an acceleration potential of $200 \mathrm{kV}$. Field emission scanning electron microscopy (FE-SEM) was also used for complimentary imaging through the use of a JEOL-JSM-7100F instrument. Steady state photoluminescence (PL) spectroscopy was measured on samples mounted with carbon tape using a Nikon Optiphot Fluorescence microscope with a Hg lamp and excitation 
filter centered at $370 \mathrm{~nm}$ and interfaced to an Ocean Optics spectrometer. Photoluminescence quantum efficiency (PLQE) was measured according to published procedures $^{42}$ using an integrated sphere from Newport using a laser excitation wavelength of $405 \mathrm{~nm}$ at $18 \mathrm{~mW} \mathrm{~cm}$ for $\mathrm{MAPbBr}_{3}$ and $532 \mathrm{~nm}$ at $10 \mathrm{~mW} \mathrm{~cm}$ for $\mathrm{MAPbI}_{3}$. For a given sample, PLQE was measured in air after samples were exposed to a modest $\left(10^{-2}\right.$ torr $)$ vacuum overnight to minimize the impact of oxygen in these measurements; samples were kept in a desiccator when not being evaluated. Powder Xray diffraction (XRD) patterns were collected with a using a Bruker SmartLab SE diffractometer equipped with a HyPix-400 detector.

\section{Supporting Information}

Supporting Information is available from the Wiley Online Library or from the author.

\section{Acknowledgements}

This work was supported by the Robert A. Welch Foundation (Grant P-1212 to JLC). S. D. S. acknowledges support from the Royal Society and Tata Group (UF150033). GD acknowledges the Royal Society through a Newton International Fellowship and the EPSRC (EP/R023980/1) for funding. K.F. acknowledges a George and Lilian Schiff Studentship, Winton Studentship, the Engineering and Physical Sciences Research Council (EPSRC) studentship, Cambridge Trust Scholarship, and Robert Gardiner Scholarship.

\section{References}

[1] W. S. Yang, B.-W. Park, E. H. Jung, N. J. Jeon, Y. C. Kim, D. U. Lee, S. S. Shin, J. Seo, E. K. Kim, J. H. Noh, S. I. Seok, Science 2017, 356, 1376. 
[2] J.-P. Correa-Baena, A. Abate, M. Saliba, W. Tress, T. Jesper Jacobsson, M. Gräzzel, A. Hagfeldt. Energy Environ. Sci. 2017, 10, 710-727.

[3] N. Aeineh, E. Barea, A. Behjat, N. Sharifi, I. Mora-Sero. ACS Appl. Mater. Interfaces. 2017, 9, 13181-13187.

[4] S. Stranks, H. Snaith, Nat. Nanotechnol. 2015, 10, 391-402.

[5] J.-P. Correa-Baena, M. Saliba, T. Buonassisi, M. Grätzel, A. Abate, W. Tress, A. Hagfeldt. Science. 2015, 358, 739-744.

[6] B. Zhao, S. Bai, V. Kim, R. Lamboll, R. Shivanna, F. Auras, J. Richter, L. Yang, L. Dai, M. Alsari, X.-J. She, L. Liang, J. Zhang, S. Lilliu, P. Gao, H. Snaith, J. Wang, N. Greenham, R. Friend, D. Du. Nature Photonics, 2018, 12, 783-789.

[7] Q. Zhang, M. Tavakoli, L. Gu, D. Zhang, L. Tang, Y. Gao, J. Guo; Y. Lin, S.-F. Leung, S. Poddar, Y. Fu, Z. Fan. Nature Communications, 2019, 10 (1), 727.

[8] S. Veldhuis, P. Boix, N. Yantara, M. Li, T. Sum, N. Mathews, S. Mhaisalkar, Adv. Mater. 2016, 28, 6804-6834.

[9] B. Sutherland, E. Sargent. Nat. Photonics. 2016, 10, 295-302.

[10] Z. Xiao, R. Kerner, L. Zhao, N. Tran, K. Lee, T.-W. Koh, G. Scholes, B. Rand. Nat. Photonics. 2017, 11, 108-115.

[11] M. Yuan, L. Quan, R. Comin, G. Walters, R. Sabatini, O. Voznyy, S. Hoogland, Y. Zhao, E. Beauregard, P. Kanjanaboos, Z. Lu, D. Kim, E. Sargent. Nature

Nanotechnology. 2016, 11, 872

[12] N. Wang, L. Cheng, R. Ge, S. Zhang, Y. Miao, W. Zou, C. Yi, Y. Sun, Y. Cao, R. Yang, Y. Wei, Q. Guo, Y. Ke, M. Yu, Y. Jin, Y. Liu, Q. Ding, D. Di, L. Yang, G. Xing, H. Tian, C. Jin, F. Gao, R. Friend, J. Wang, W. Huang. Nature Photonics. 2016, 10, 699

[13] National Renewable Energy Laboratory, Best research-cell efficiencies (accessed May 2020); www.nrel.gov/pv/cell-efficiency.html.

[14] N.-G. Park, Materials Today 2015, 18, 65-72.

[15] S. D. Stranks, G. E. Eperon, G. Grancini, C. Menelaou, M. J. P. Alcocer, T. Leijtens, L. M. Herz, A. Petrozza, H. J. Snaith, Science 2013, 342, 341.

[16] D. P. McMeekin, G. Sadoughi, W. Rehman, G. E. Eperon, M. Saliba, M. T. Hörantner, A. Haghighirad, N. Sakai, L. Korte, B. Rech, M. B. Johnston, L. M. Herz, H. J. Snaith, Science 2016, 351, 151.

[17] A. Miyata, A. Mitioglu, P. Plochocka, O. Portugall, J. T.-W. Wang, S. D. Stranks, 
H. J. Snaith, R. J. Nicholas, Nature Physics 2015, 11, 582-587.

[18] L. M. Herz, ACS Energy Letters 2017, 2, 1539-1548.

[19] C. Gehrmann, D. A. Egger, Nature Communications 2019, 10, 3141.

[20] S. Shao, M. A. Loi, Advanced Materials Interfaces 2020, 7, 1901469.

[21] A. Loiudice, S. Saris, E. Oveisi, D. Alexander, R. Buonsanti. Angewandte Chemie International Edition. 2017, 56 (36), 10696-10701.

[22] J. Burschka, N. Pellet, S.-J. Moon, R. Humphry-Baker, P. Gao, M. Nazeeruddin, M. Grätzel. Nature 2013, 499, 316.

[23] K. Lim, C. Deakin, B. Ding, X. Bai, P. Griffin, T. Zhu, R. Oliver, D. Credgington, APL Materials. 2019, 7, 021107.

[24] D. Dirin, L. Protesescu, D. Trummer, I. Kochetygov, S. Yakunin, F. Krumeich, N. Stadie, M. Kovalenko. Nano Letters. 2016, 16, 5866-5874.

[25] R. Gonzalez-Rodriguez, N. Arad-Vosk, A. Sa'ar, J. Coffer. Journal of Physical Chemistry C. 2018, 122, 20040-20045.

[26] N. Arad-Vosk, N. Rozenfeld, R. Gonzalez-Rodriguez, J. Coffer, A. Sa'ar. Phys. Rev. B. 2017, 95,085433.

[27] R. Gonzalez-Rodriguez, N. Arad-Vosk, N. Rozenfeld, A. Sa'ar, J. Coffer. Small. 2016, 12, 4477-4480.

[28] R. Gonzalez-Rodriguez, V. C. P. Costa, G. Delport, K. Frohna, R. L. Z. Hoye, S. D. Stranks, J. L. Coffer, Nanoscale 2020, 12, 4498-4505.

[29] T. Leijtens, K. A. Bush, R. Prasanna, M. D. McGehee, Nature Energy 2018, 3, 828838.

[30] A. Loni, "Porous Silicon Formation by Anodization" in Handbook of Porous Silicon, $2^{\text {nd }}$ Ed, L.T. Canham, Ed., 2018, Springer, pp 13-24.

[31] J. Lauerhaas, M. Sailor, Science, 1993, 261, 1567.

[32] V. Malgras, S. Tominaka, J. Ryan, J. Henzie, T. Takei, K. Ohara, Y. Yamauchi. Journal of the American Chemical Society. 2016, 138, 13874-13881

[33] A. Sadhanala, F. Deschler, T. H. Thomas, S. E. Dutton, K. C. Goedel, F. C. Hanusch, M. L. Lai, U. Steiner, T. Bein, P. Docampo, D. Cahen, R. H. Friend, Journal of Physical Chemistry Letters 2014, 5, 2501-2505. 
[34] N. Droseros, G. Longo, J. C. Brauer, M. Sessolo, H. J. Bolink, N. Banerji, ACS Energy Letters 2018, 3, 1458-1466.

[35] R. Brenes, D. Guo, A. Osherov, N. K. Noel, C. Eames, E. M. Hutter, S. K. Pathak, F. Niroui, R. H. Friend, M. S. Islam, H. J. Snaith, V. Bulović, T. J. Savenije, S. D. Stranks, Joule 2017, 1, 155-167.

[36] M. Anaya, J. F. Galisteo-López, M. E. Calvo, J. P. Espinós, H. Míguez, Journal of Physical Chemistry Letters 2018, 9, 3891-3896.

[37] R. Brenes, C. Eames, V. Bulović, M. S. Islam, S. D. Stranks, Advanced Materials 2018, 30, 1706208.

[38] Y. Tian, M. Peter, E. Unger, M. Abdellah, K. Zheng, T. Pullerits, A. Yartsev, V. Sundström, I. G. Scheblykin, Physical Chemistry Chemical Physics 2015, 17, 2497824987.

[39] S. Demchyshyn, J. M. Roemer, H. Groiß, H. Heilbrunner, C. Ulbricht, D. Apaydin, A. Böhm, U. Rütt, F. Bertram, G. Hesser, M. C. Scharber, N. S. Sariciftci, B. Nickel, S. Bauer, E. D. Głowacki, M. Kaltenbrunner, Science Advances 2017, 3, e1700738.

[40] E. Yablonovitch, T.J. Gmitter, R. Bhat. Physical Review Letters 1988, 61, 25462549 .

[41] M. M. Byranvand, A. N. Kharat, N. Taghavinia, Materials Letters 2019, 237, 356360 .

[42] A. Loni, "Gas Adsorption Analysis of Porous Silicon" in Handbook of Porous Silicon, $2^{\text {nd }}$ Ed, L.T. Canham, Ed., 2018, Springer, pp 593-599.

[43] S. Leyre, E. Coutino-Gonzalez, J. J. Joos, J. Ryckaert, Y. Meuret, D. Poelman, P. F. Smet, G. Durinck, J. Hofkens, G. Deconinck, P. Hanselaer, Review of Scientific Instruments 2014, 85, 123115. 


\section{Table of Contents Entry}

Metal halide perovskites, and the devices constructed from them, are famously vacancy tolerant. By changing template pore diameter and associated surface chemical composition to modulate interfacial defects and properties, this work addresses the corresponding impact on perovskite luminescence wavelength, intensity, and stability.

\section{Perovskites}

Impact of Mesoporous Silicon Template Pore Dimension and Surface Chemistry on

\section{Methylammonium Lead Trihalide Perovskite Photophysics}

V.C.P. da Costa, R. Gonzalez-Rodriguez, K. Frohna, G. Delport, S.D. Stranks, L.T.

Canham, J.L. Coffer

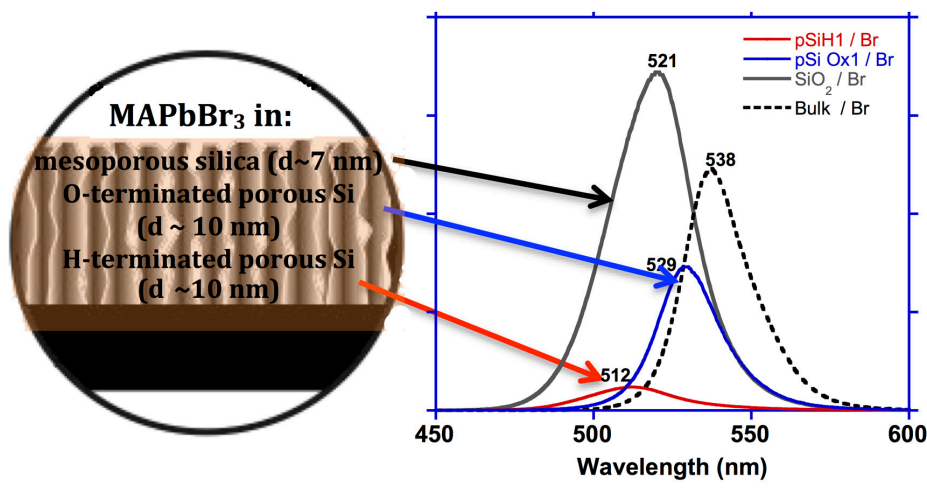

